\title{
THE EFFECT OF TRABECULECTOMY ON REFRACTION, KERATOMETRY AND CORNEAL TOPOGRAPHY
}

\author{
K. G. CLARIDGE, J. K. GALBRAITH, V. KARMEL and A. K. BATES \\ Taunton
}

\begin{abstract}
SUMMARY
After successful trabeculectomy patients often complain of reduction in vision even after several months. Amongst other factors, corneal astigmatism appears to be altered. A pilot study measuring, pre- and postoperative corneal topography indicated three types of astigmatic change: some patients develop a relative superior corneal steepening, others a superior flattening and yet others complex regional changes that do not conform to either of these patterns. The present study was designed to evaluate further these patterns of variation in corneal curvature and to look for corresponding refractive and keratometry changes. Twenty-nine patients admitted for trabeculectomy had pre-operative assessment of subjective and automated refraction, manual keratometry and corneal topography from which simulated keratometry values were calculated. A standard trabeculectomy procedure was performed and post-operative measurements of the same parameters were taken at 1 and 3 months after surgery. Similar patterns of corneal topographic change to those found in our pilot study were noted. In both the superior steepening and superior flattening groups there was an increase in vertical keratometry and a shift towards 'with-the-rule' astigmatism. Furthermore a 1 year analysis of 13 patients from our pilot study indicated that the topographic changes lasted for at least 12 months after surgery. We conclude that computer-assisted corneal topography reveals complex regional changes in corneal curvature that are not readily detected from alterations in refraction or keratometry. These changes are sufficiently great to have a significant effect on visual function in some patients.
\end{abstract}

Over the last few years several studies have suggested that early trabeculectomy may be superior to medical therapy in the treatment of primary

From: Department of Ophthalmology, Taunton and Somerset Hospital Trust, Musgrove Park, Taunton, Somerset TA1 5DA. Correspondence to: Ms K. G. Claridge, MA, DO, FRCOphth, Bristol Eye Hospital, Lower Maudlin Street, Bristol BS1 2LX, UK. Tel: (0117) 923006. Fax: (0117) 9251421. open angle glaucoma. ${ }^{1,2}$ Although this course of action may benefit the control of glaucoma, some studies have indicated that surgery affects corneal curvature and therefore may lead to a deterioration in visual acuity. ${ }^{3-6}$ This is of particular importance as many patients undergoing trabeculectomy have excellent pre-operative vision.

Cunliffe et al. ${ }^{4}$ measured changes in refraction and keratometry in 16 patients undergoing trabeculectomy. They found there was a post-operative myopic shift which correlated with the shallowing of the anterior chamber, lasting about 3 weeks. Their keratometry data confirmed that of Hugkulstone ${ }^{3}$ and showed a shift towards 'with-the-rule' astigmatism which returned to the pre-operative value by 10 months after surgery. Rosen et al. ${ }^{5}$, using computerassisted corneal topography, measured a postoperative steepening in the vertical axis, and found that in many cases this steepening was greater than that suggested by keratometry.

A pilot study was carried out in this hospital by two of the authors (J.K.G. and A.K.B.) in 1992. Twenty patients were enrolled and computerised corneal topography was performed before and after trabeculectomy. Three types of change emerged: some subjects showed a superior corneal steepening, whereas others developed a superior corneal flattening; yet other subjects had complex regional changes which did not conform to either of the two previous groups.

Having found that trabeculectomy had these varied effects on corneal topography, a study was designed to investigate whether the changes observed were associated with corresponding, clinically relevant, variations in refraction and keratometry.

\section{PATIENTS AND METHODS}

Twenty-nine patients were recruited who had primary open angle glaucoma which was not adequately controlled with medical therapy. In no 
case had there been previous ocular surgery, other than laser trabeculoplasty, and no patient had any other ocular pathology.

All subjects were admitted for trabeculectomy which was performed by a number of surgeons using a standard technique. This consisted of a fornixbased conjunctival flap, a $4 \times 3 \mathrm{~mm}$ partial-thickness scleral flap under which a full-thickness block of clear cornea was excised. Two 10-0 nylon sutures were placed at the corners of the scleral flap and three 8-0 vicryl sutures to the conjunctiva. Topical antibiotics and atropine were given. Post-operatively all patients had a standard regime of topical steroid, antibiotic and mydriatic. No subject had a serious complication and at 3 months all had satisfactory intraocular pressure control (mean $13.0 \mathrm{mmHg}$; $95 \%$ confidence interval $11.6-14.4 \mathrm{mmHg}$ ), though in 2 cases this required the continued use of a topical beta blocker.

At the pre-operative assessment and at 1 and 3 months post-operatively each patient was examined as follows: visual acuity (Snellen Acuity Chart), subjective refraction, automated refraction (Canon AutoRef R-22, taking the average of three readings), manual keratometry (Bausch and Lomb) and computer-assisted corneal topography (Topographic Modelling System TMS-1). From this last measurement topography was expressed as a colour-coded contour map. A simulated keratometry reading was calculated from the maximum meridional powers of rings 6,7 and 8 . The average of the maximum powers, the axis at which this occurred and the average power of the corneal surface for the same rings at the meridian $90^{\circ}$ away were recorded.

Astigmatic variation involves a change in both magnitude and orientation of refractive cylinder and it can be difficult to express quantitatively. Polar values, described by Naeser in $1990,{ }^{7}$ calculate the balance between 'with-the-rule' and 'against-therule' components of any given astigmatism. They therefore enable corneal astigmatism to be expressed as a single figure. An increase in polar value indicates a shift towards 'with-the-rule' astigmatism whereas a decrease indicates a move towards 'against-the-rule' astigmatism. Polar values were calculated for the net astigmatism measured by both manual and simulated keratometry.

Measurements from only one eye of each subject were used in the study. Longitudinal variation with time for a given parameter was assessed by both Analysis of Variance (ANOVA) for Repeated Measurements and Wilcoxon's signed rank test for non-parametric data, unless otherwise stated. Statistical significance was accepted if $p<0.05$ for either test.

\section{RESULTS}

When the 1 month post-operative corneal topo- graphic map was compared with the pre-operative map for each patient, similar patterns of change to those described in the pilot study were noted. The characteristics of each of the three groups are shown in Table I.

Some patients developed a superior corneal steepening. Fig. 1 illustrates this pattern of postoperative change as a differential display map. The pre-operative (B) and 1 month post-operative (A) corneal topographic maps are shown on the left with the dioptric values expressed by the left-hand colour scale. On the right is the difference surface map in which the pre-operative picture $B$ has been subtracted from the post-operative picture $\mathrm{A}$, with the dioptric values represented by the right-hand colour scale. This patient had a superior steepening of almost 1 dioptre at the 1 month assessment. Overall 14 patients showed this pattern of change. Their mean superior steepening was 1.1 dioptres at 1 month and 1.0 dioptres at 3 months after surgery (Table II).

Other subjects developed a superior corneal flattening as shown in Fig. 2. In this case the flattening measured 0.7 dioptre at 1 month after surgery. The mean flattening for the 5 eyes displaying this pattern of change was 0.8 dioptre at 1 month and 0.7 dioptre at 3 months post-operatively (Table II).

There was a significant difference in the change of the superior corneal curvature between the superior steepening and superior flattening groups (at 3 months: ANOVA for factor analysis $p=0.0001$, Mann-Whitney $U p<0.003$ ).

Finally, in 10 of the 29 subjects there were complex regional variations in corneal topography which did not fit any particular pattern (Fig. 3). These subjects could not be allocated to either the superior steepening or superior flattening groups. There was no difference in the average age, the number of right and left eyes, or the surgeon when the three groups were compared.

Having identified these patterns of post-operative topographic alteration within the superior cornea, the refraction and keratometry data were examined for comparable changes (Table II). The superior steepening group developed a myopic shift, measured by both subjective and automated refraction, which lasted at least 3 months after surgery though significance was not reached. The manual keratometry data revealed a significant increase in the horizontal and vertical keratometry values after surgery (horizontal $K$ : ANOVA repeated measurements $p<0.007$, Wilcoxon's $p<0.009$ at 3 months; vertical $K$ : ANOVA repeated measurements $p<0.0002$, Wilcoxon's $p<0.002$ at 3 months). Although the polar value showed a shift towards 'with-the-rule' astigmatism this was not significant. There were similar changes in the calculated 
Table I. Characteristics of corneal topography subgroups

\begin{tabular}{lccc}
\hline & Superior steepening & Superior flattening & Irregular change \\
\hline Number & 14 & 5 & 10 \\
Male : female & $6: 3$ & $5: 0$ & $6: 4$ \\
Right : left eye & $10: 4$ & $2: 3$ & $5: 5$ \\
Mean age (years) $(95 \%$ CIs) & $70.2(63.0,75.4)$ & $69.4(64.2,74.7)$ & $74.1(66.0,82.1)$ \\
\hline
\end{tabular}

simulated keratometry. Only the increase in vertical keratometry and polar value was significant (vertical $K$ : ANOVA repeated measurements $p<0.05$, Wilcoxon's $p<0.006$ at 3 months; polar value: Wilcoxon's $p<0.05$ at 3 months but ANOVA repeated measurements $p>0.05$ ).

The superior flattening group did not show any particular refractive change. The manual keratometry data indicated an increase in both horizontal and vertical keratometry values though only the change in the vertical $K$ value was significant at 3 months (Wilcoxon's $p<0.05$ but ANOVA repeated measurements $p>0.05)$. The polar value showed no consistent variation. The data from the simulated keratometry showed a similar increase in the vertical keratometry value though the change was not significant. However, there was an increase in the polar value suggesting a shift towards 'with-the-rule' astigmatism which was significant at 3 months (Wilcoxon's $p<0.05$ but ANOVA repeated measurements $p>0.05$ ).

There was good correlation between the spherical equivalent measured by subjective and automated refraction respectively (Spearman's rank correlation $p=0.0001)$ and between the horizontal and vertical keratometry values measured by manual and simulated keratometry respectively (Spearman's rank correlation $p=0.0001$ for both comparisons). The change in visual acuity measured pre-operatively and at 3 months post-operatively is illustrated in Fig. 4. Most subjects maintained their pre-operative acuity and in those who showed a deterioration this was not associated with any particular pattern of corneal topographical change. More sophisticated tests of visual function were not assessed.

In order to investigate the duration of the observed changes in corneal curvature 13 patients from the pilot study were examined at 1 year after surgery. Six subjects from the superior steepening group showed a persistent topographic change with a group mean steepening of 1.08 dioptres (95\% CI 0.73 to 1.45 ) at 1 year. The mean change for the 7 subjects in the superior flattening group did not suggest a persistent change (mean superior corneal flattening -0.24 dioptre, $95 \% \mathrm{CI}-0.60$ to 1.08 ) though some subjects showed a flattening of 1 dioptre at the 1 year assessment.

Amongst the subjects whose corneal topographic changes could not be allocated to the superior steepening or flattening groups were individuals who developed an isolated steepening or flattening in the central corneal region (Figs. 5 and 6 respectively). At the 1 year analysis subject 4 has a central steepening of 1.5 dioptres compared with the pre-operative picture and subject 5 demonstrated a 1.5 dioptre difference when comparing the superior and inferior corneal curvature.

\section{DISCUSSION}

Computer-assisted corneal topography confirms that corneal curvature is affected by trabeculectomy. There appear to be two broad patterns of topographic variation, the most common being a postoperative steepening of the corneal curvature

Table II. Group mean values (95\% confidence intervals) for refractive parameters for the superior steepening and superior flattening groups

\begin{tabular}{|c|c|c|c|c|c|c|}
\hline & \multicolumn{3}{|c|}{ Superior steepening group } & \multicolumn{3}{|c|}{ Superior flattening group } \\
\hline & Pre-op. & 1 month & 3 months & Pre-op. & 1 month & 3 months \\
\hline $\begin{array}{l}\text { Subjective refraction } \\
\text { Spherical equivalent }\end{array}$ & $-0.1(-2.1,+1.9)$ & $-0.6(-2.5,+1.3)$ & $-0.7(-2.5,+1.1)$ & $-0.5(-4.5,+3.6)$ & $-0.2(-3.6,+3.3)$ & $-0.6(-4.6,+3.4)$ \\
\hline $\begin{array}{l}\text { Autorefraction } \\
\text { Spherical equivalent }\end{array}$ & $+0.1(-1.9,+2.0)$ & $-0.2(-2.1,+1.7)$ & $-0.2(-1.9,+1.6)$ & $-0.4(-4.2,+3.4)$ & $-0.1(-3.6,+3.3)$ & $-0.6(-4.6,+3.3)$ \\
\hline $\begin{array}{l}\text { Manual keratometry } \\
\text { Horizontalkeratometry } \\
\text { Vertical keratometry } \\
\text { Polar value }\end{array}$ & $\begin{array}{r}42.9(41.8,44.0) \\
43.3(42.2,44.4) \\
0.4(-0.4,1.1)\end{array}$ & $\begin{array}{rr}43.7 & (42.5,44.9) \\
44.5 & (43.5,45.6) \\
0.6 \quad(0.1,1.2)\end{array}$ & $\begin{array}{r}43.8(42.8,44.9) \\
44.6(43.5,45.6) \\
0.7(-0.7,1.4)\end{array}$ & $\begin{array}{r}42.8(40.6,45.0) \\
43.0(41.5,44.5) \\
0.2(-1.0,1.4)\end{array}$ & $\begin{array}{l}43.6(41.7,45.6) \\
43.3(41.7,44.9) \\
-0.3(-0.8,0.2)\end{array}$ & $\begin{array}{r}43.7(42.2,45.2) \\
43.8(41.8,45.7) \\
0.1(-0.5,0.7)\end{array}$ \\
\hline $\begin{array}{l}\text { Simulated keratometry } \\
\text { Horizontal keratometry } \\
\text { Vertical keratometry } \\
\text { Polar value }\end{array}$ & $\begin{array}{r}43.9(42.7,44.1) \\
44.6(43.5,45.7) \\
0.6(-0.2,1.3)\end{array}$ & $\begin{array}{r}44.1(43.0,45.3) \\
45.2(44.3,46.1) \\
1.0(0.4,1.6)\end{array}$ & $\begin{array}{r}44.3(43.1,45.5) \\
45.2(44.0,46.4) \\
1.0(0.1, \quad 1.8)\end{array}$ & $\begin{array}{l}44.3(42.0,46.7) \\
43.9(41.5,46.4) \\
-0.3(-1.0,0.4)\end{array}$ & $\begin{array}{r}44.4(42.1,46.7) \\
44.3,(41.9,46.7) \\
0.1(-1.1, \quad 1.2)\end{array}$ & $\begin{array}{l}44.3(42.4,46.2) \\
44.4(42.0,46.8) \\
0.0(-0.8,0.8)\end{array}$ \\
\hline Superior corneal chang & & $1.1 \quad(0.9,1.2)$ & $1.0(0.6,1.3)$ & & $-0.8(-1.6,0.0)$ & $-0.7(-1.6,0.2)$ \\
\hline
\end{tabular}

All measurements are in dioptres. 


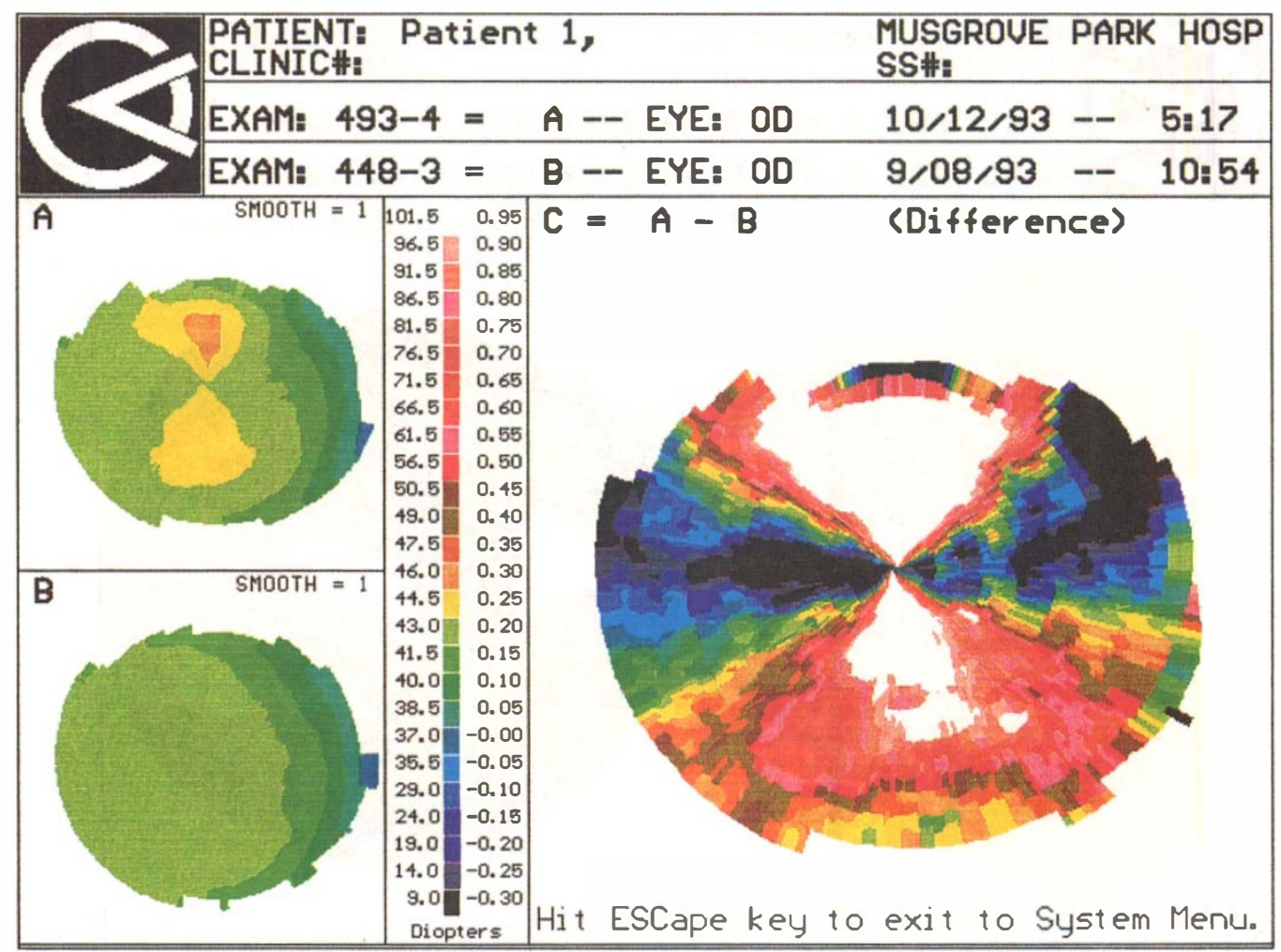

Fig. 1. One month post-operative difference display corneal topography map of a subject developing superior corneal steepening.

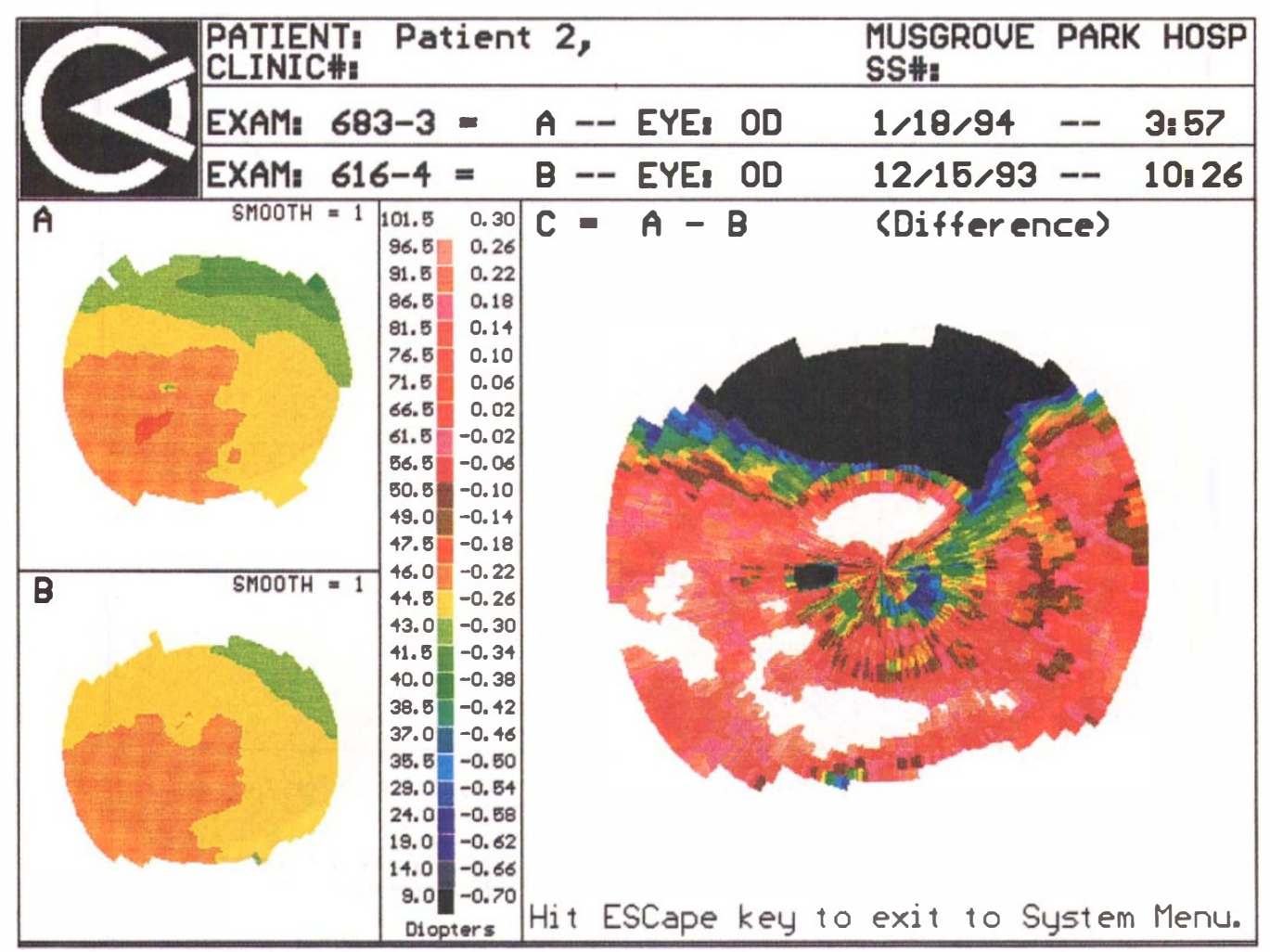

Fig. 2. One month post-operative difference display corneal topography map of a subject developing superior corneal flattening. 


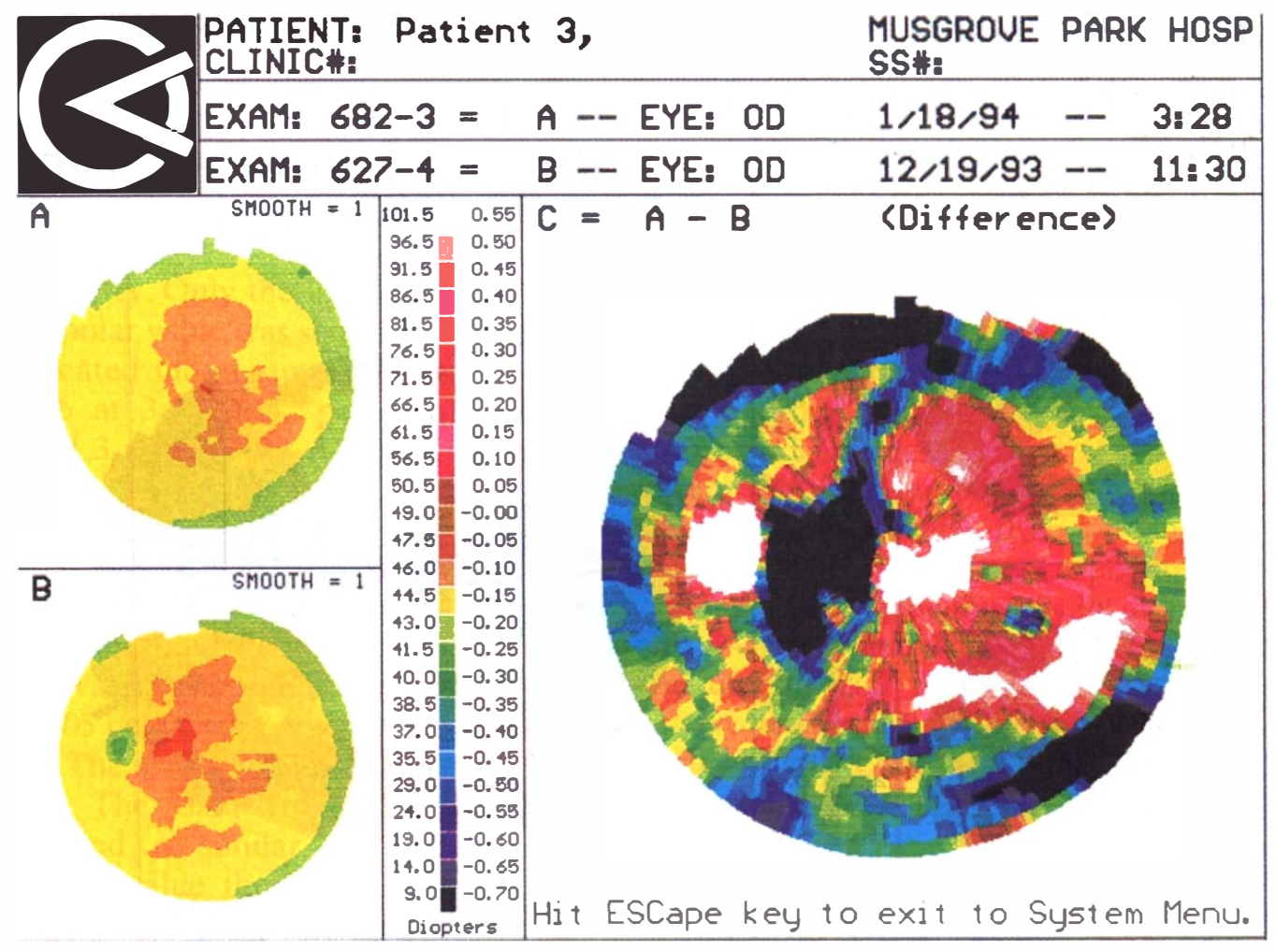

Fig. 3. One month post-operative difference display corneal topography map of a subject developing complex regional changes.

pre-operative visual acuity

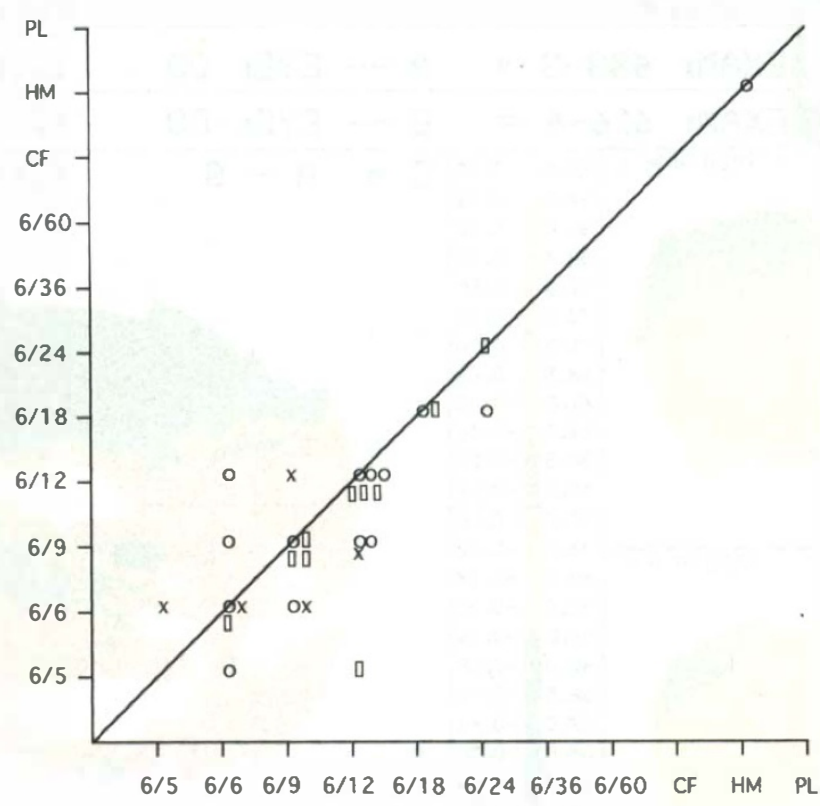

visual acuity at 3 months post-operative

Fig. 4. Pre-and post-operative Snellen visual acuity measurements. Circles, patients from superior steepening group ( $\mathrm{n}=14)$; crosses, patients from the superior flattening group $(\mathrm{n}=5)$; rectangles, patients who showed complex regional changes $(n=10)$. 


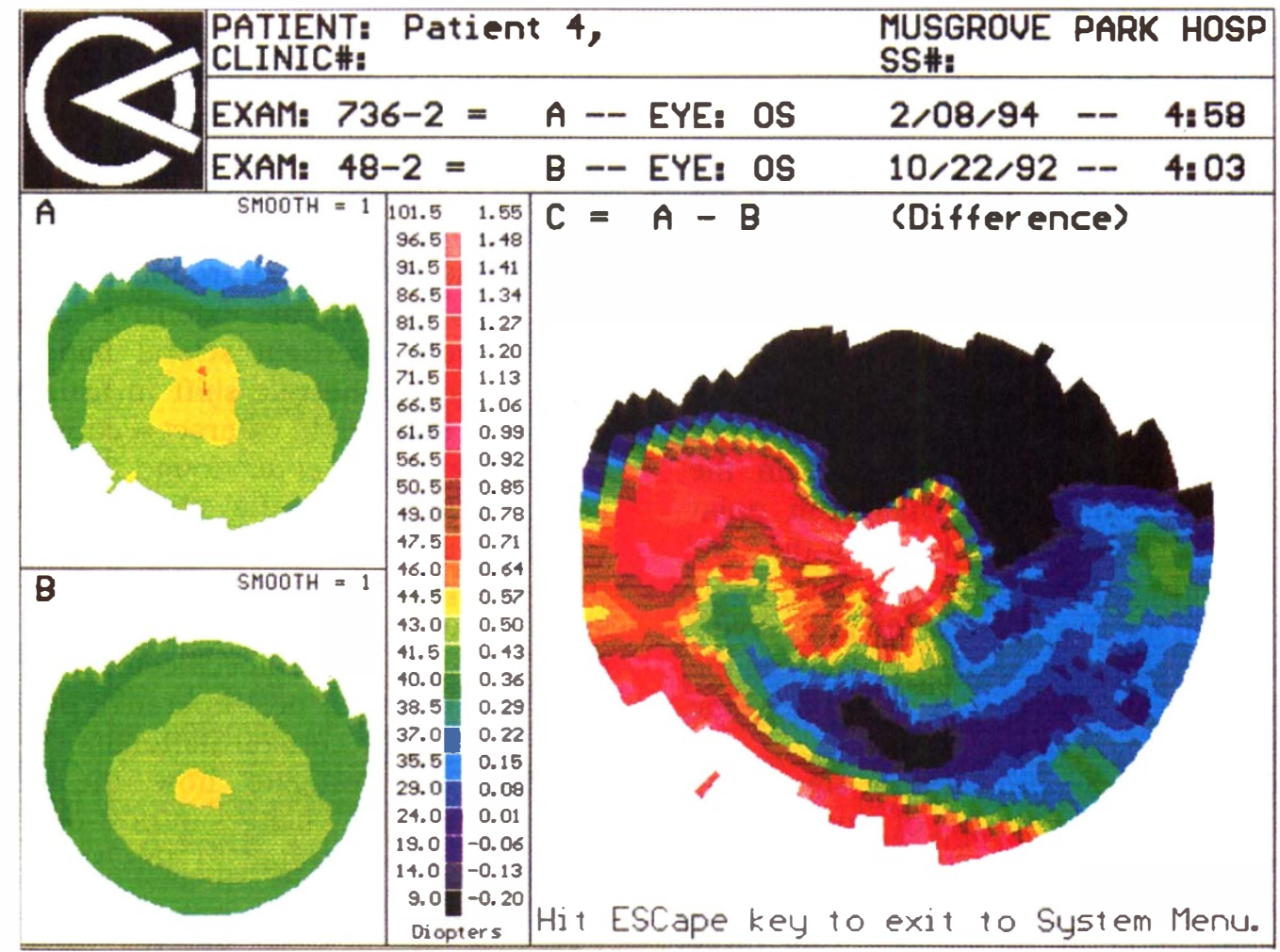

Fig. 5. One year post-operative difference display corneal topography map of a subject showing central corneal steepening.

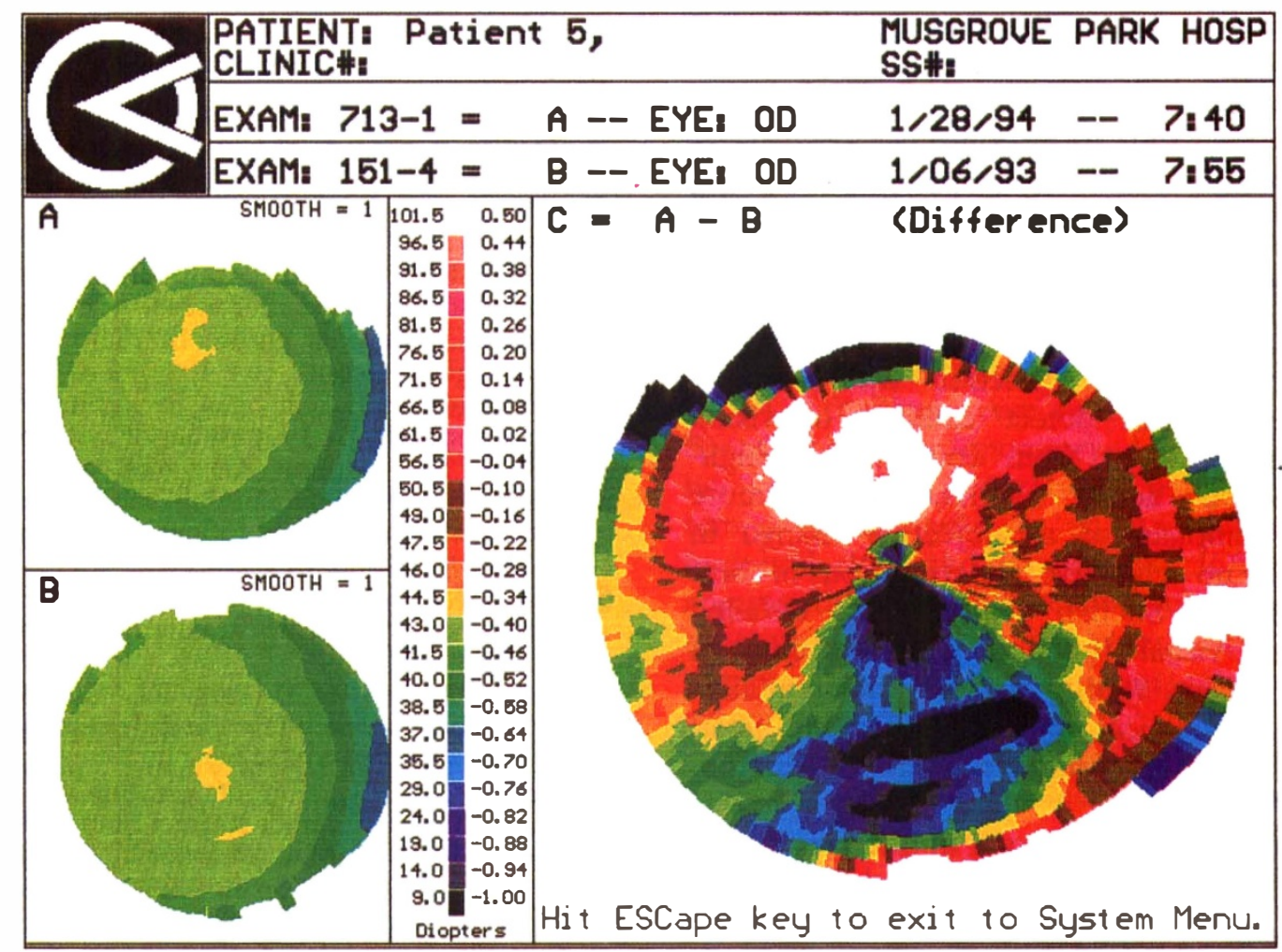

Fig. 6. One year post-operative difference display corneal topography map of a subject showing central corneal flattening. 
although a smaller number of patients developed a superior corneal flattening.

The associated changes in refractive and keratometry data were complex and did not readily differentiate the two groups. In both groups there was a consistent trend towards an increase in the vertical keratometry reading. One might expect the superior flattening group to develop a decrease in the post-operative vertical keratometry reading. However, as shown in Fig. 2, several subjects developed an inferior steepening coexistent with the superior flattening, resulting in an overall increase in the vertical keratometry value. The change in polar value indicated a shift towards 'with-the-rule' astigmatism in both groups. This was compatible with the change in vertical keratometry, implying that both groups develop an overall steepening in the vertical meridian which occurred in either the superior or inferior region of the cornea.

Classification of patients into superior steepening and superior flattening patterns of topographic change probably oversimplifies what seems to be a complex regional change in corneal curvature and there were many different variations within these two broad categories. Furthermore an isolated steepening or flattening of the central cornea would be expected to affect vision as much as a change in superior or inferior corneal curvature by inducing an irregular astigmatism, uncorrectable by sphericocylindrical lenses.

Our data from Snellen acuity measurements indicated that only a minority of patients showed a post-operative deterioration in vision. However, Rosen et al..$^{5}$ found that of 6 patients with induced post-operative with-the-rule astigmatism, 4 lost at least one line of Snellen acuity. More sophisticated measurements of visual function with contrast sensitivity testing can detect changes in vision which are not evident from conventional highcontrast Snellen acuity charts. ${ }^{8}$ Furthermore patients do complain of a subjective change in vision despite no objective deterioration in best spectacle-corrected Snellen acuity. More detailed assessment of visual function with low-contrast sensitivity testing as well as patient questionnaires may clarify the clinical importance of the postoperative changes in corneal topography demonstrated.

In contrast to the findings of Cunliffe et al. ${ }^{4}$ the results from our pilot study indicated that in some subjects the observed change in corneal curvature lasted for over a year. The magnitude of these changes was sufficiently great in some patients to be expected to have a significant effect on vision.

The alteration in corneal topography may be related to several components of trabeculectomy surgery. A superior corneal steepening may arise from tissue contraction around the trabeculectomy site as a result of extensive scleral cautery. Alternatively overtight scleral flap sutures could lead to a with-the-rule shift in astigmatism in the same way that a tight cataract section causes corneal steepening. Rosen et $a l^{5}$ found that suture lysis did not prevent astigmatic change whereas scleral cautery was associated with corneal steepening in the post-operative period. If the scleral flap is loose, superior corneal flattening may develop from wound gape around the trabeculectomy site. A large drainage bleb or a post-operative ptosis could also lead to superior corneal flattening. It would be of interest to investigate how the above factors correlate with the changes in post-operative corneal curvature demonstrated in this study, in a larger series of patients.

We should like to thank Mr J. M. Twomey and Mr R. H. Gray for allowing us to study their patients. The Corneal Topographer was purchased by a generous grant from the Friends of Musgrove Hospital.

Key words: Corneal topography, Keratometry, Refraction, Trabeculectomy.

\section{REFERENCES}

1. Jay JL. Rational choice of therapy in primary open angle glaucoma. Eye 1992;6:243-7.

2. Migdal C. Rational choice of therapy in established open angle glaucoma. Eye 1992;6:346-7.

3. Hugkulstone CE. Changes in keratometry following trabeculectomy. Br J Ophthalmol 1991;75:217-8.

4. Cunliffe IA, Dapling RB, West J, Longstaff S. A prospective study examining the changes in factors that affect visual acuity following trabeculectomy. Eye 1992;6:618-22.

5. Rosen WJ, Mannis MJ, Brandt JD. The effect of trabeculectomy on corneal curvature. Ophthalmic Surg 1992;23:395-8.

6. Dietze PJ, Koch DD, Gross RL. Corneal topography after trabeculectomy [abstract]. Invest Ophthalmol Vis Sci 1992;33(Suppl 6):2343.

7. Naeser K. Conversion of keratometry readings into polar values. J Cataract Refract Surg 1990;16:741-5.

8. Elliott DB, Hurst MA, Weatherill J. Comparing clinical tests of visual function in cataract with the patient's perceived visual disability. Eye 1990;4:712-7. 